according to the serum levels of LDL-C; Groups 1, 2, 3 consisted of hypolipidemic patients $(n=61)$, normolipidemic patients $(n=135)$ and hyperlipidemic patients $(n=161)$, respectively. HOMA-IR above 3.42 was defined as IR.

Body mass index were not different among these groups $(\mathrm{p}$ $>0.05)$. The frequency of NAFLD was $44.5 \%$ in the entire study group and were $52 \%, 44.7 \%$ and $41.4 \%$ in the Groups 1,2 , 3, respectively. The frequency of NAFLD were not different among these three groups ( $p>0.05$ ). In NAFLD patients, HDL-C levels were lower than in patients without NAFLD $(p>0.01)$ and negatively correlated with ALT levels $(\mathrm{r}=-0.1, \mathrm{p}<0.05)$.

In patients with IR $(n=172)$, the frequency of NAFLD and triglyceride levels were higher and HDL-C levels were lower than in patients without IR $(n=178)$. The frequency of IR were not different among the Groups 1,2 and 3 ( $\mathrm{p}>0.05$ ).

It is found out that hypolipidemic obese patients, clinically not given much attention, have NAFLD at least as frequent as hyperlipidemic patients do. On the other hand, obese patients with IR and NAFLD have atherogenic profile; high trygliyceride and low HDL-C levels. Low HDL-C levels may be related with also hepatosteotitis in the NAFLD. Therefore, abnormalities in lipid metabolism are significant contributers of obesity and associated co-morbidities.

\section{P529 RECOGNITION OF OBESITY IN CHILDREN ATTENDING A PAEDIATRIC EMERGENCY DEPARTMENT (THE ROIC STUDY)}

Jeanne Cloonan*, Emer Dwyer, Eimear Carlos, Ireti Farombi. Our Lady of Lourdes Hospital, Drogheda, Ireland

\subsection{6/archdischild-2019-epa.864}

Background Childhood obesity is a global public health problem, linked to adult obesity and its associated co-morbidities. A study in a Paediatric Emergency Department in London in the BMJ in 2014 found a low recognition and referral rate for Paediatric Obesity. ${ }^{1}$ However, no such studies have been performed to date in Ireland.

Aims To ascertain the incidence of obesity in patients attending our Paediatric Emergency Department.

To establish recognition of obesity by paediatric medical staff.

Methods This was a Prospective Study. Nursing staff were asked to measure heights along with weights at triage of children between 2 and 16 years of age, over a one week period. Inclusion criteria were paediatric medical patients, aged 2-16 yrs. Surgical/trauma patients, those who's height had not been documented and those who did not wait to be seen were excluded.

BMI centiles were calculated on all those meeting inclusion criteria. In those who plotted as overweight or obese, age, gender, presenting complaint, co-morbidities and nutritional history, if taken, were documented, along with whether the raised BMI was recognised/documented by medical staff and if they received a referral for further follow-up.

Results A search of the Emergency Department IPMS system found 222 patients aged 2-16 years attended the Paediatric Emergency Department during the week of the study.

The ED charts of 149 patients who met criteria were reviewed. Heights were recorded in 61/149 (40\%). BMI centiles were plotted for those patients and 20/61 (33\%) were found to be overweight or obese.

Of these patients, 10 were overweight (between $91^{\text {st }}$ and $98^{\text {th }}$ Centile) and 10 were obese ( $>98^{\text {th }}$ Centile). $1 / 20$ patients $(5 \%)$ was noted to be obese and was referred to a dietician. $12(60 \%)$ were male and $8(40 \%)$ were female.

The most common presenting complaints were abdominal pain $4(20 \%)$ and chest pain $3(15 \%)$.

$5 / 20(25 \%)$ had other documented co-morbidities.

Conclusions These results show a low recognition of paediatric obesity in the Emergency Department by paediatric medical staff.

These results also show a high incidence of obesity in our population however as only $40 \%$ of patients had heights measured the true incidence is difficult to determine.

These results support the need for further education of paediatric staff in the recognition of obesity and the development of guidelines and an appropriate referral pathway.

\section{REFERENCE}

1. Knight M, Booth C. Obesity management in a Paediatric Emergency Department BMJ Qual Improv Rep. 2014;3(1):u203067.w1454.

\section{P530 ASSOCIATIONS BETWEEN DISORDERED EATING ATTITUTES AND BEHAVIORS IN ADOLESCENTS WITH OBESITY AND DEPRESSION AND ANXIETY}

${ }^{1}$ Aslı Okbay Güneş*, ${ }^{2}$ Müjgan Alikaşifoğlu, ${ }^{3}$ Ezgi Şen Demirdöğen, ${ }^{4}$ Ethem Erginöz, ${ }^{3}$ Türkay Demir, ${ }^{5}$ Oya Ercan. ${ }^{1}$ Istanbul University-Cerrahpaşa, Department of Pediatrics, Istanbul, Turkey; ${ }^{2}$ stanbul University-Cerrahpaşa, Department of Pediatrics, Division of Adolescent Medicine, Istanbul, Turkey; ${ }^{3}$ Istanbul University-Cerrahpaşa, Department of Child and Adolescent Mental Health and Diseases, Istanbul, Turkey; ${ }^{4}$ Istanbul UniversityCerrahpaşa, Department of Public Health, Istanbul, Turkey; ${ }^{5}$ Istanbul University-Cerrahpaşa, Department of Pediatrics, Division of Adolescent Medicine and Endocrinology, Istanbul, Turkey

\subsection{6/archdischild-2019-epa.865}

Objective The aim of this study was to determine whether there were any associations between disordered eating attitudes and behaviors and depression and anxiety among obese adolescents.

Methods A prospective cross-sectional study comprising 80 obese adolescents was performed from November 2013 to September 2014. Disordered eating attitudes were evaluated by the 'Eating Disorder Examination Questionnaire (EDE-Q)' and 'Dutch Eating Behavior Questionnaire(DEBQ)'. 'Eating Disorder Examination Questionnaire' contains four subscales that evaluate restraint, eating concerns, shape concerns, and weight concerns, and the items evaluate eating attitudes of the individual over the last four weeks. 'Dutch Eating Behavior Questionnaire' comprises three subscales which measure restrained eating, emotional eating, and external eating behaviors. Psychiatric examinations were performed for binge eating disorder (BED). Depression was evaluated by 'Children's Depression Inventory (CDI)', and anxiety was evaluated by the 'State-Trait Anxiety Inventory for Children'. Mann-Whitney U, Chi-Square and Pearson Correlation tests were used for statistical analysis.

Results Mean age of the subjects was $14.01 \pm 1.59$ years. Forty-six $(57.5 \%)$ of the subjects were girls. Mean body mass index was found as $31.29 \pm 3.06 \mathrm{~kg} / \mathrm{m}^{2}$. Twenty adolescents were found to have depression and 23 adolescents were found to have BED. There were significant associations between 